\title{
Investigations of Residual Stresses and Mechanical Properties of Single Crystal Niobium for SRF Cavities
}

\author{
Thomas Gnäupel-Herold ${ }^{1}$, Ganapati Rao Myneni ${ }^{2}$, Richard E. Ricker ${ }^{3}$ \\ ${ }^{1}$ National Institute of Standards and Technology, Center for Neutron Research, 100 Bureau Dr., \\ Gaithersburg, MD 20899, USA and University of Maryland, Dept. of Materials Science and \\ Engineering, College Park, MD 20742-2115 \\ ${ }^{2}$ Thomas Jefferson National Accelerator Facility, Accelerator Division, 12000 Jefferson Avenue, \\ Newport News, VA 23606 \\ ${ }^{3}$ National Institute of Standards and Technology, Metallurgy Division, 100 Bureau Dr., \\ Gaithersburg, MD 20899
}

\begin{abstract}
This work investigates properties of large grained, high purity niobium with respect to the forming of superconducting radio frequency (SRF) cavities from such large grained sheets. The yield stresses were examined using tensile specimens that were essentially single crystals in orientations evenly distributed in the standard projection triangle. No distinct yield anisotropy was found, however, vacuum annealing increased the yield strength by a factor 2..3. The deep drawing forming operation of the half cells raises the issues of elastic shape changes after the release of the forming tool (springback) and residual stresses, both of which are indicated to be negligible. This is a consequence of the low yield stress ( $<100 \mathrm{MPa})$ and the large thickness (compared to typical thicknesses in sheet metal forming). However, the significant anisotropy of the transversal plastic strains after uniaxial deformation points to potentially critical thickness variations for large grained / single crystal half cells, thus raising the issue of controlling grain orientation or using single crystal sheet material.
\end{abstract}

\section{Introduction}

The promise of performance increases from using large grained / single crystal (1-10 grains per cell) niobium SRF cavities over finer grained polycrystalline cavities ( $>10^{10}$ grains per cell) has initiated an intense research effort with the goal to identify critical properties in the production and use of such cavities. The cells of the cavities are formed by means of deep drawing from a flat, circular sheet. Due to the bending/unbending processes during deep drawing a throughthickness plastic strain differential is created which induces residual stresses. Their magnitude depends on the yield stress, the accumulated plastic strain, and the hardening behavior. The ability of the material to accumulate large strains is closely related to formability. Thus, areas of critical interest for assessing the feasibility of large grained SRF cavities are formability and the closely related topics springback and residual stresses. Due to the small number of constituent grains the forming is dominated by single crystal behavior which, with respect to the deep drawing operation, is largely unknown. Established means for characterizing formability such as the determination of the forming limit diagram, yield surface determination using biaxial deformation tests such as punch test or Marciniak-type balanced biaxial stretching are either not applicable (because deep drawing of SRF cavities has no significant stretching) and/or are prohibitively expensive due to high material cost and the need for a representative range of orientations, which, in the initial sheet, are random and not controlled in the production. With 
respect to the limited amount of sample material it was concluded that uniaxial tensile tests are best suited to characterize forming related properties of plastic deformation. In order to elucidate springback and residual stresses a series of non-destructive and destructive tests was performed. Non-destructive tests were done using neutron and X-ray diffraction. Slicing tests were used to measure deflections upon release of forming related residual stress.

\section{Experimental}

\section{Orientations}

The as-received material was a circular disk with a diameter of $233 \mathrm{~mm}$ and a thickness of $3.09 \mathrm{~mm}$. The surface was treated by buffered chemical etching. The arrangement of the grains and their normal orientations are shown in Fig. 1. The orientations were determined by means of $\mathrm{X}$-ray diffraction in a four-circle goniometer.

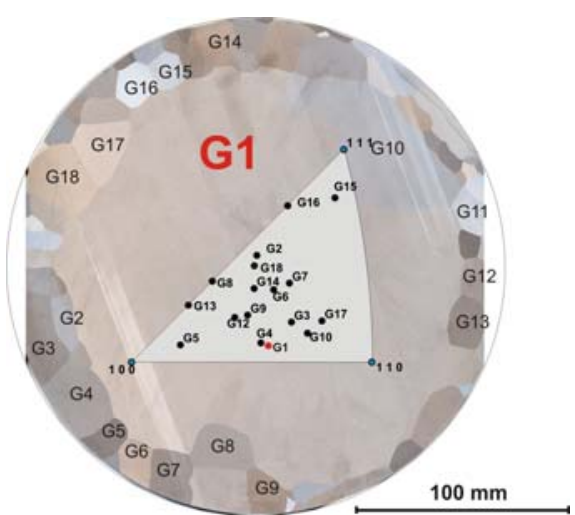

Figure 1. Scanned image of the as-received disk. The different grains are clearly visible with the large central grain taking about $2 / 3$ of the total area.

The grain orientations appear to be random with no clear relationship between the orientation of the large central grain and its neighbors. This large spread enabled the extraction of tensile samples with orientations evenly spread out in standard projection (Fig. 2).
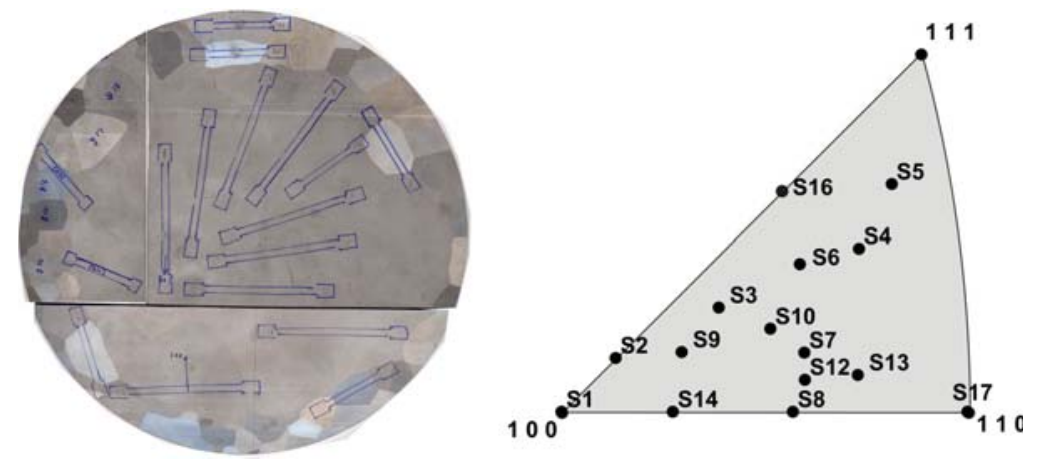

Figure 2. Arrangement of tensile samples on the initial disk with their respective orientations for the tensile axis (same disk as in Fig. 1). Two different lengths of tensile samples were used; the choice of length depended on the size of the grain; it was set such that the gage section was as uniform in orientation as possible. The standard projection on the right shows the tensile axis orientations. 


\section{Tensile Tests}

An initial set of tensile tests was performed on electro-discharge machine (EDM) cut specimens without further treatment. EDM cutting was performed under de-ionized water. The results are shown in Fig. 3.
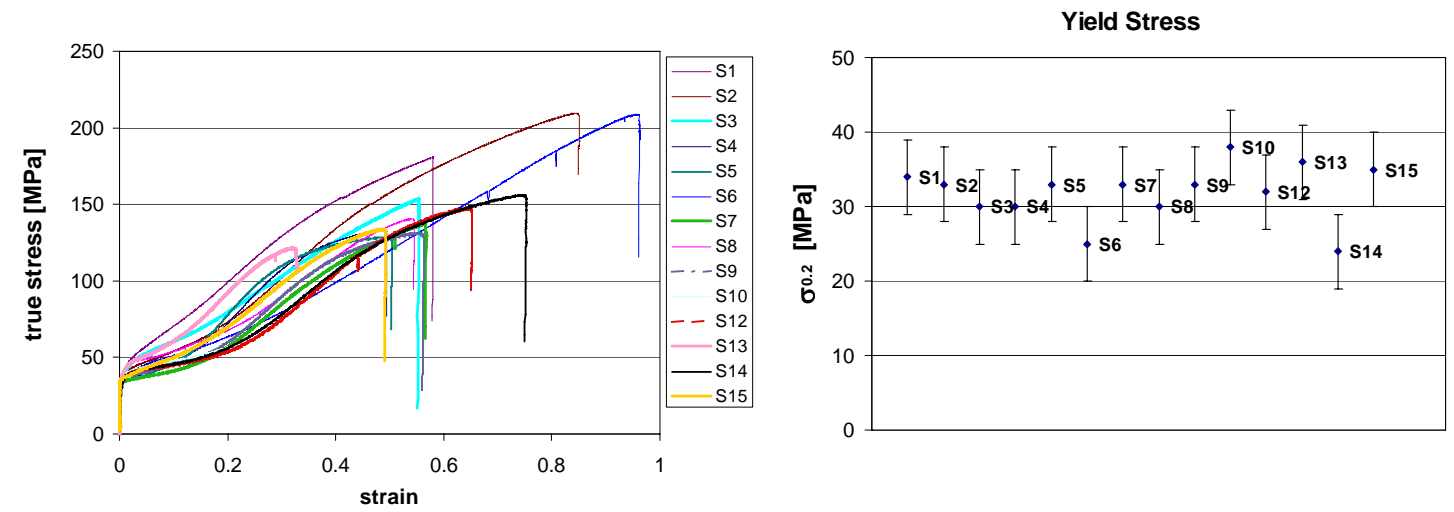

Figure 3. Stress-strain curves for uniaxial tensile deformation in the orientations shown in Fig. 2. The yield stresses shown on the right were derived from the data shown on the left as stress value at $0.2 \%$ strain.

With a typical uncertainty of $0.5 \%$ for the strain measurement the resulting uncertainty for the yield stress determination is $\pm 5 \mathrm{MPa}$. The yield stresses do not exhibit a statistically significant anisotropy outside this range. The tensile experiments were performed at constant speed, which, due to different gage lengths, lead to different strain rates of $10^{-4} \mathrm{~s}^{-1}$ or smaller. The relationship between strain rate and yield stress is shown in Fig. 4.

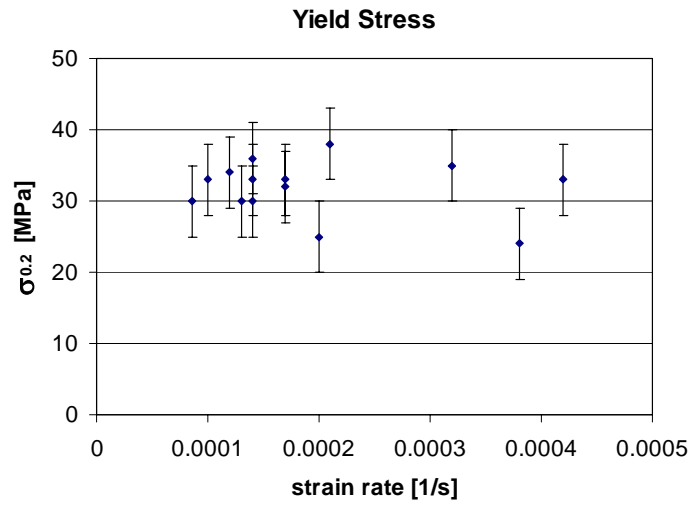

Figure 4. Yield stress vs. strain rate.

There is no statistically significant rate sensitivity found in Fig. 4. However, this is due to the fact that all yield stress variations occur within $1 \times \sigma$ and the possibility of anisotropy effects. It should also be noted that the softness of the specimens caused a visible effect of the extensometer clamping force (rubber band) on the deformation of the tensile samples with visible shape distortions in the region of the extensometer knife edges. It is therefore possible that both the yield stress and the strain at failure were negatively affected by the extensometer clamping. However, with failure strains generally $>50 \%$ up to $100 \%$ it is estimated that the extensometer clamping contributed only in a minor way to the low yield stresses. Some specimens exhibited a yield drop with no obvious connection to orientation with differences in the upper and lower yield point of $<1 \mathrm{MPa}$ (Fig. 3). All stress-strain curves showed typical single crystal behavior with 
stage I/II/III hardening [1]; however, the proportions of the hardening stages are not uniform. Both the absence/presence of the yield drop and differences in hardening behavior cannot be clearly attributed to anisotropy and are likely affected by sample inhomogeneities as well. Overall, the yield stresses are substantially lower than reported elsewhere [2]. The comparison of specimen preparation procedures lead to the hypothesis of hydrogen absorption during the water immersed EDM cutting. This prompted a second series of tests in which additional specimen were EDM cut with subsequent vacuum annealing. The purpose of these tests was a thorough outgassing of volatile interstials which are known to affect the mechanical properties of high purity niobium [3].

The first annealing test was done for 1 hour at $700^{\circ} \mathrm{C}$ at $10^{-6} \mathrm{Torr}$; the second test with a different set of samples was done for 24 hours at $1000{ }^{\circ} \mathrm{C}$. The results of the tensile tests are shown in Fig. 5.
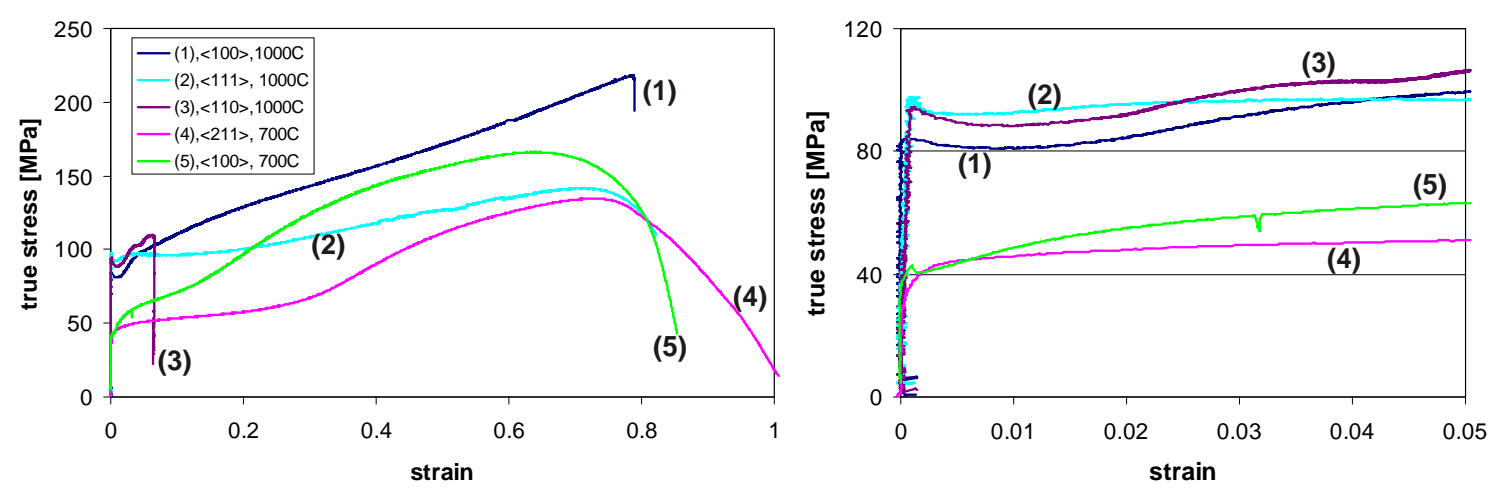

Figure 5: Stress-strain curves after annealing. The figure on the right side shows the first $5 \%$ strain in detail. The strain rate was $10^{-5} \mathrm{~s}^{-1}$ for all annealed samples.

The first annealing treatment resulted in a moderate increase of the yield stress to $41 \mathrm{MPa}$ both for the $<211>$ and the $<100>$ orientation. The annealing stage at $1000^{\circ} \mathrm{C}$ raises the yield point approximately by a factor of three with values of $84 \mathrm{MPa}(<100>), 94 \mathrm{MPa}(<110>)$ and $97 \mathrm{MPa}$ $(<111>$ ). A notable difference to the initial tensile tests is the low failure strain ( $<10 \%$ strain) for the $<110>$ oriented sample which failed at a grain boundary near the gage section. Also, all samples annealed at $1000^{\circ} \mathrm{C}$ exhibit a yield drop of approximately $5 \mathrm{MPa}$ which is more pronounced than in the specimens without annealing.

Formability

A basic descriptor for formability is the R-value which is a measure of the resistance against thinning during deep drawing. As shown in Fig. 6, it is defined as the ratio of the transversal shrinking strain to the thickness reduction strain and it can range from 0 (thinning only) to values $>1$ (little thinning).

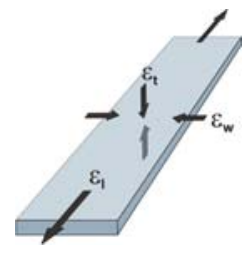

$$
R=\frac{\varepsilon_{w}}{\varepsilon_{t}}
$$

Figure 6. Definition of the R-value. 
The R-values derived from the tensile tests are shown in Fig. 7.

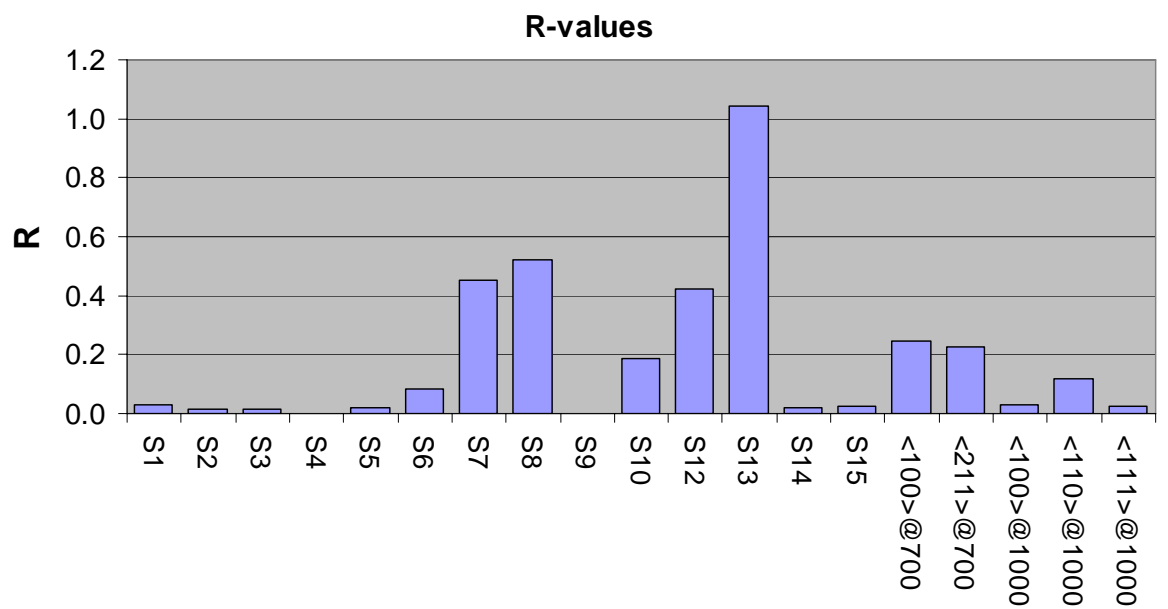

Figure 7. R-values as measured from the reduction ratios of the tensile samples.

The largest R-values are found for specimen orientations clustered around a $<210>$ direction (specimen S13 has an orientation close to $<320>$ ). For body centered cubic niobium a $<111>$ slip plane is expected with slip possible directions of the type $\{211\}$ and $\{011\}$. A Schmid factor analysis shows that for all specimens the resolved shear stress is maximized with $\langle 111)\{2 \overline{1} \overline{1}\}$ as slip system. Large R-factors are found for specimen orientations in which the slip direction is perpendicular to the surface direction of the disk from which the specimens were cut. However, the large R-factor discrepancy between samples identical in orientation and cut from the same grain but different in the annealing treatment (S1, <100>@700 $\left.{ }^{\circ} \mathrm{C},<100>@ 1000^{\circ} \mathrm{C}\right)$ shows that there is some probability for other active slip systems.

It should be noted that the initial rectangular character of the specimen cross section was not retained during tensile testing but, depending on orientation, more or less distorted into a parallelogram. Thus, the transversal shrinking strain had to calculated using the measured thickness and width measured at half thickness. The parallelogram distortion is the result of slip directions that are between the surface normal direction and the in-plane direction of the tensile specimens.

Dimensional Analysis

The dimensional analysis was performed by scanning the inner and outer surface of a half cell (Fig. 8) using a coordinate measuring machine.

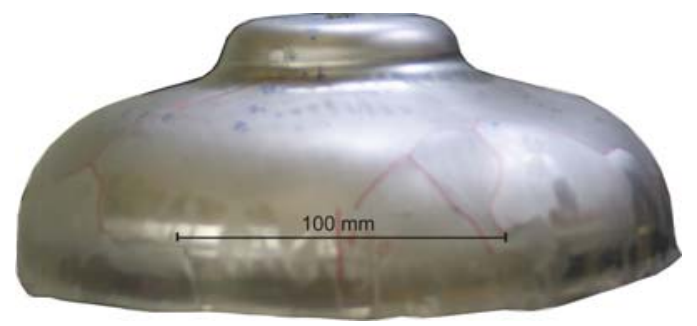

Figure 8. Large grained niobium SRF half cell. Grain Boundaries are clearly visible near the open bottom. 
The analysis provides quantitative measures for the extent of thinning (effect of the anisotropic Rvalue) as well as the effect of grain boundaries on shape variations. The thinning affects the roundness (i.e. the radii taken perpendicular to the vertical symmetry axis of the half cell in Fig. 8) and the wall thickness while the effect of grain boundaries is strain localization which leads to sudden increases or decreases in thickness. This is shown in Fig. 9-11. The most severe localization effects are found near the equator (Fig. 11) before trimming. However, this region is subject to removal before the welding of the cells; therefore, a more accurate measure of localization effects is Fig. 9 (roundness at bottom ) which shows a roundness distribution that is slightly less smooth that the roundness in the single crystal region. A measure of local thinning is shown in Fig. 10. These data were taken after trimming of the equator region. The peripheral grains intrude into the region from $-40 \mathrm{~mm}$ to $-55 \mathrm{~mm}$ (vertical), which is clearly reflected in the thickness variations due to different thinning of individual grains.

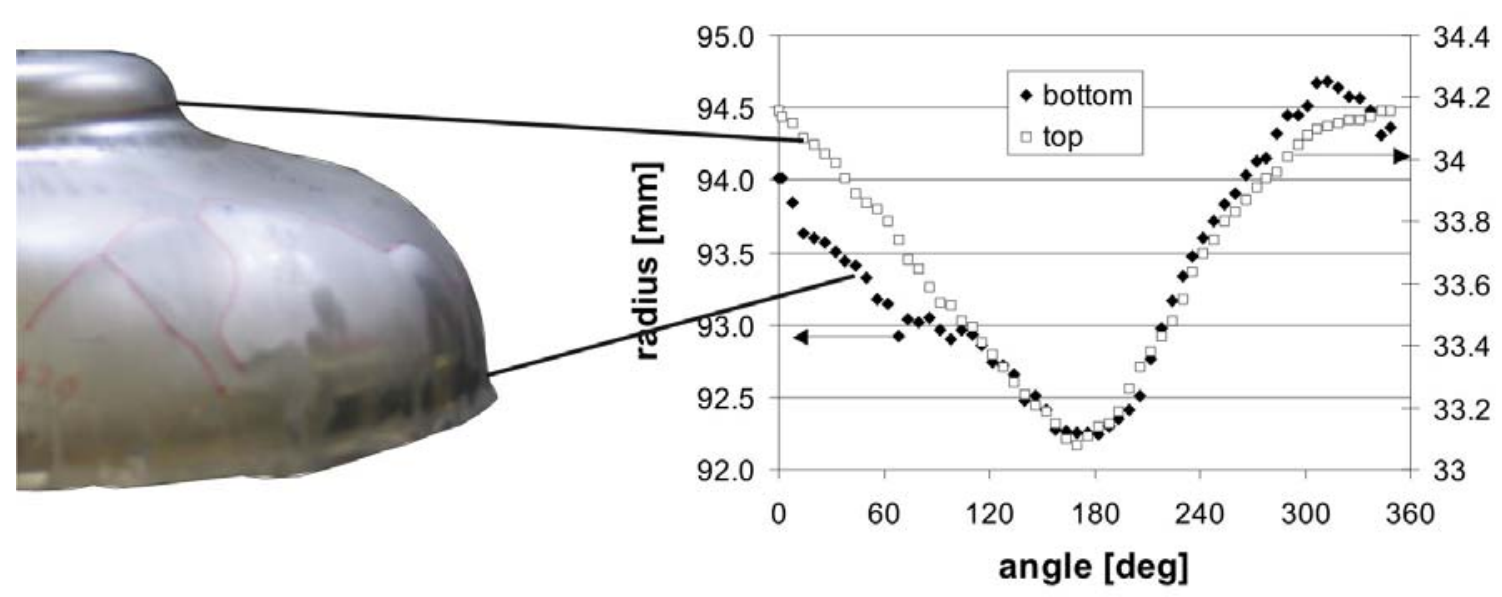

Figure 9. Roundness of the half cell as measured at two vertical positions. The error is $\pm 0.01 \mathrm{~mm}$.
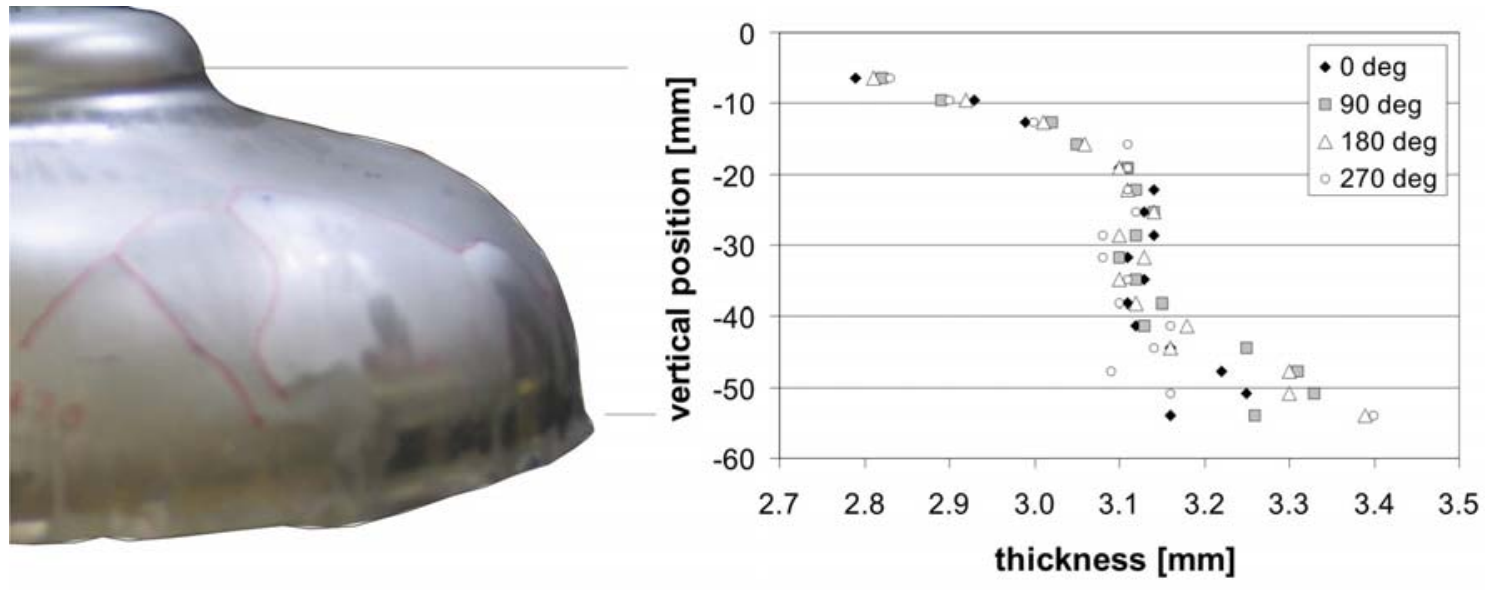

Figure 10. Thickness variations as measured in $90 \mathrm{deg}$. intervals. The error is $\pm 0.02 \mathrm{~mm}$. 


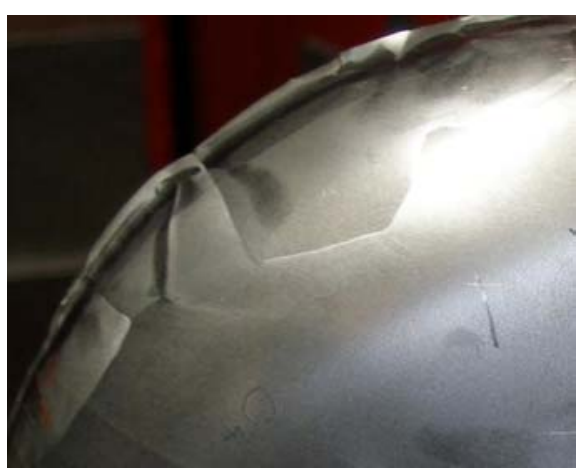

Figure 11. Strain localization near a grain boundary.

\section{Residual Stresses}

The destructive evaluation of stresses involve a series of EDM cuts for sectioning the specimens in different orientations with the purpose of relieving/equilibrating certain stress components. It should be noted that here, strictly speaking, stresses are not completely relieved but allowed to balance in certain directions after which the resulting bending moment is zero. These techniques have a narrow scope (typically good for only one stress component per cutting orientation with multiple specimens required) but the accuracy is generally good. Only one half cell was available for testing, which required a sectioning sequence as shown in Fig. 12.
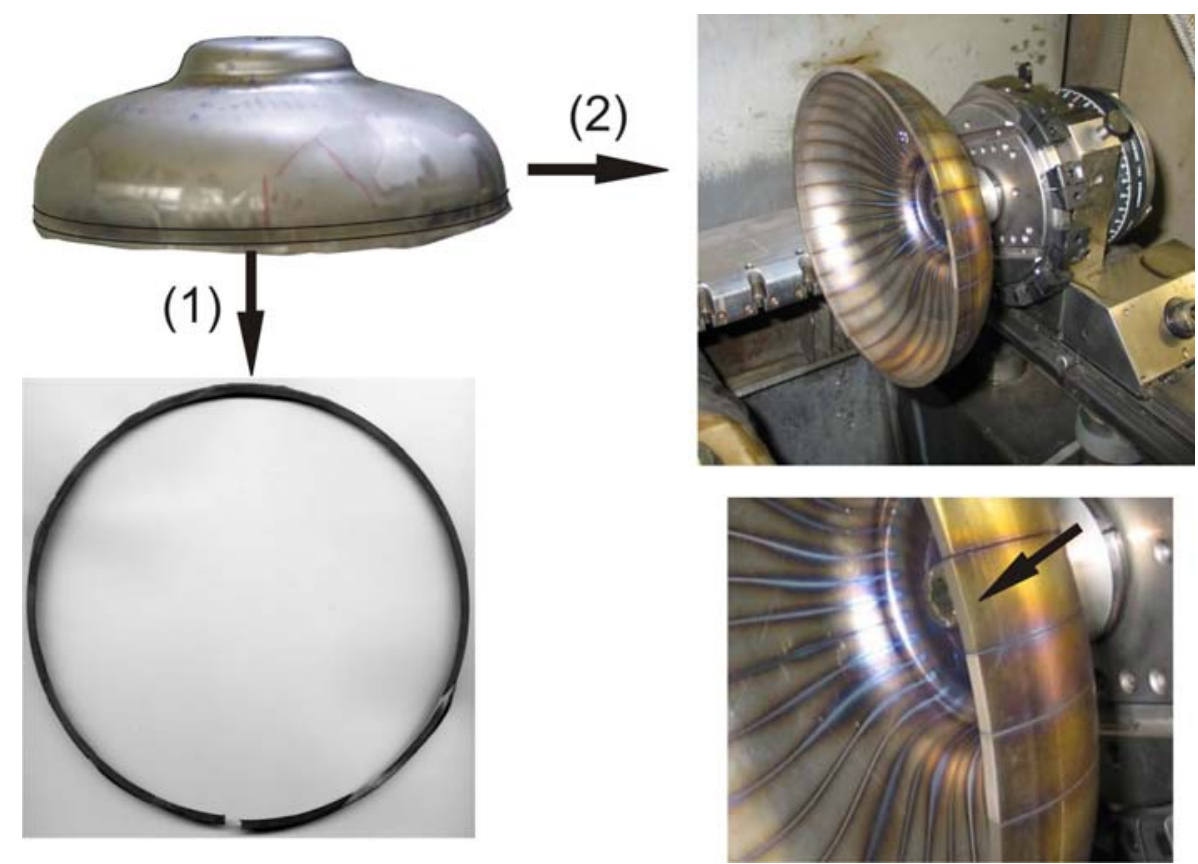

Figure 12. Slicing sequence for the release of hoop stresses (1) and radial/axial stresses (2). Stage (1) finishes with the splitting of the ring cut from the bottom. In the "flower-cut" the width of the petals in (2) is 10 degrees. The bottom right picture shows the location of the petal deflection measurement. 
The hoop stress release after ring splitting yields a stress change before/after of (23 \pm 20$) \mathrm{MPa}$ which was calculated according to [4,5]. The uncertainty is largely due to unknown orientations of grains in the peripheral region. The deflections after stage (2) are shown in Fig. 13.

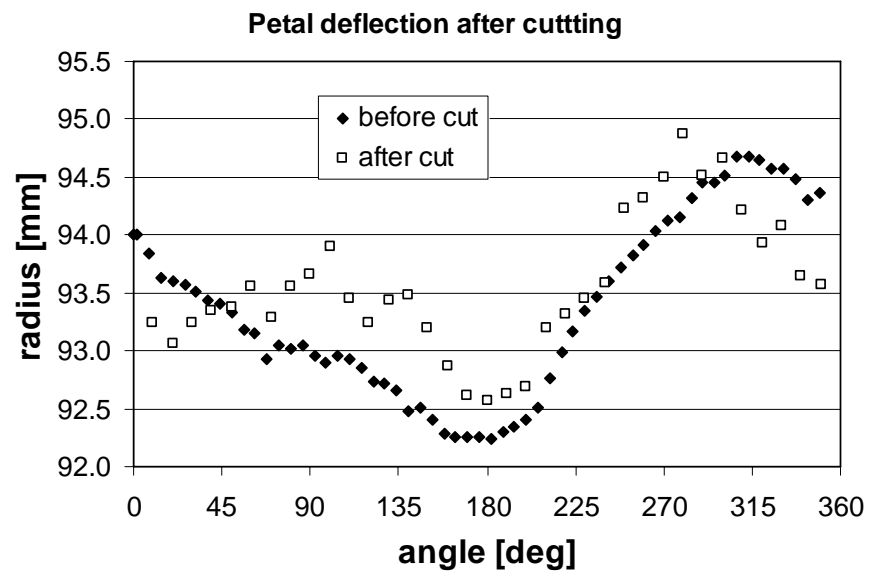

Figure 13. Radial deflections of the petals after stage (2) slicing. The radii were taken at the vertical location shown in Fig. 12. The errors are $\pm 0.01 \mathrm{~mm}$.

The deflections in Fig. 13 represent an integral effect because stresses are relieved along the entire length of the petal. Also, because of the lever rule the stresses at locations farthest away from the end of the petal (measurement location) have the largest influence on the deflection. This location is near zero for the vertical coordinate as shown in Fig. 10 which is also the location of the largest reduction in thickness.

\section{X-ray and Neutron Diffraction}

X-ray diffraction was used to determine the residual stresses both on a polycrystal half cell and a single crystal half cell. The $\sin ^{2} \psi$-technique [6] was used for the polycrystal while the single crystal method is described in [7]. The location of the measurements both for the inside and the outside is shown in Fig. 14. The results of the measurements are listed in Tab. 1.

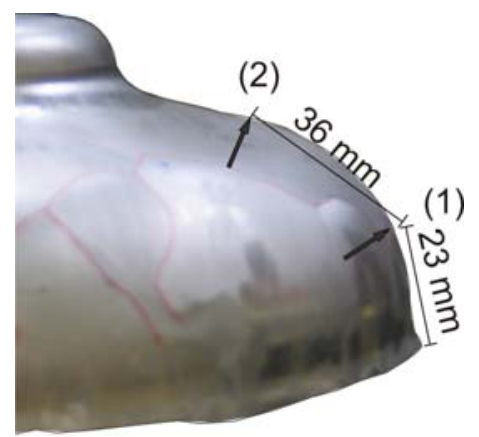

Figure 14. Location of the X-ray measurements (both inside and outside).

Table 1. Results of the X-ray stress measurements on a polycrystal and a large grained half cell. The circumferential location for the large grained sample was chosen such that both locations (1) 
and (2) were on the same grain (large central grain similar to the one in Fig. 1). The measurement uncertainty is $\pm 10 \mathrm{MPa}$.

\begin{tabular}{||c|c|c|c|c||}
\hline \multirow{3}{*}{ sample } & \multicolumn{2}{|c|}{ location } & $\begin{array}{c}\text { hoop stress } \\
{[\mathrm{MPa}]}\end{array}$ & $\begin{array}{c}\text { axial stress } \\
{[\mathrm{MPa}]}\end{array}$ \\
\hline polycrystal & inside & $(1)$ & -2 & -13 \\
\cline { 2 - 5 } & inside & $(2)$ & 16 & -32 \\
\cline { 2 - 5 } & outside & $(1)$ & -11 & -28 \\
\cline { 2 - 5 } & outside & $(2)$ & -42 & -28 \\
\hline \hline \multirow{3}{*}{ single crystal } & inside & $(1)$ & -9 & -50 \\
\cline { 2 - 5 } & inside & $(2)$ & 21 & 7 \\
\cline { 2 - 5 } & outside & $(1)$ & -10 & -34 \\
\cline { 2 - 5 } & outside & $(2)$ & 10 & -10 \\
\hline
\end{tabular}

The neutron measurements represented some challenge because only one orientation allowed a suitable positioning of the specimen with a satisfactory spatial resolution of $1 \mathrm{~mm}$ which was applied in an oblique direction of the through-thickness scan. In this approach the gage volume travels $20 \mathrm{~mm}$, thus sampling strains from distances $6 \times$ thickness. The data presented in Fig. 15 should therefore be considered only as illustration of the variability of d-spacings over such a range. However, a more detailed depth profiling is planned on small pieces cut from the half cell.
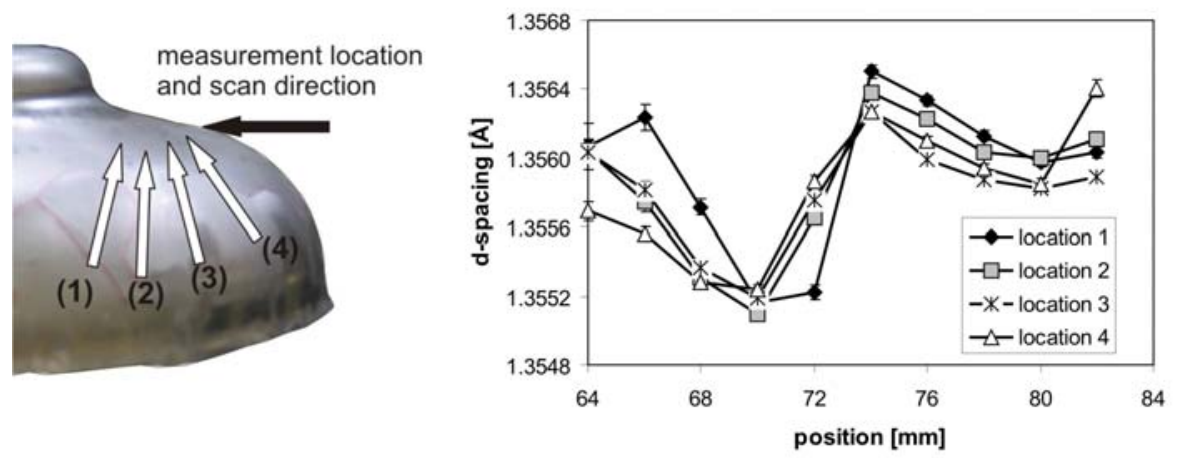

Figure 15. Inter-atomic lattice spacings measured on the large central grain at the locations shown on the left. The scan direction is indicated by the black arrow.

\section{Discussion}

Single crystal niobium shows generally a very large strain reserve in uniaxial testing with extensions of more than $80 \%$. The current forming process for the half cells has no stretching, i.e. no blank holder force, thus limiting the maximum strain during forming. It can be estimated from geometrical considerations of the blank and the cavity that strains approach or exceed $20 \%$ only locally. For example, the blank diameter is $233 \mathrm{~mm}$ while the diameter at the largest circumference of the half cell is $188 \mathrm{~mm}$. With a central outline of about $260 \mathrm{~mm}$ one estimates the strains involved at the equator to be $\approx 20 \%$ compressive and $\approx 10 \%$ tensile along the equator-iris-equator outline. Even with the biaxial nature of the forming strains these limits are safely within the margin established by the tensile tests. However, the anisotropic R-value provides indication that there is a potential for local thinning and shape deviations. Neighboring grains with different thinning behaviors can show pronounced strain localizations at their boundaries. Also, the low yield stress and the moderate anisotropy in yield and hardening should give a low potential for tearing. 
The effect of the annealing treatments is very strong and the yield stress after annealing are among the highest reported for single crystal niobium. It also illustrates the extreme sensitivity of the yield stress to interstitial impurities. Such sensitivity does not appear in the R-values after annealing.

The large grained half cell exhibits substantial variations in roundness $( \pm 1 \mathrm{~mm})$ and thickness (10 \%) in the periphery region (bottom). This is a consequence of the fact that this region consists of approximately 40-50 individual grains, each with different R-values for the active strain directions. Strain localization leads to radial variations up to $1 \mathrm{~mm}$ from one grain to the next (Fig. 11). The potential problem of increased part rejection can be eliminated if the number of grains is further reduced as reported elsewhere [8].

The investigation of the residual stress showed very small magnitudes which are at or just above the detection limit of the methods used. This finding is a result first of the low yield stresses regardless of orientation, and second of the comparatively low strain magnitudes during forming. Also, the stress-strain curves exhibit low hardening rates which result in small stress differentials in the specimen after forming. With respect to the observation of shape deviations it can be concluded that residual stresses do not contribute significantly because they are simply too low. For example, the deflections of the petals after "flower-cutting" is smaller than the roundness variations. The through-thickness stress differential is so small that there is essentially no change of bending moment. With respect to the application at superconducting temperatures, no change in the stresses is expected at working temperatures $<4 \mathrm{~K}$ due to the minor increase in elastic stiffnesses of $\approx 7 \%$.

\section{Acknowledgements}

This work is supported in part by DOE contract DE-AC05-84ER40150 and Reference Metals Company Inc. CRADA 2004-S002-Mod 2. The authors acknowledge discussions with Mr. Tadeu Carneiro and help of Dr. P. Kneisel with the large grain niobium half cells.

\section{References}

[1] R.W. Cahn, P. Haasen (Eds.), Physical Metallurgy, North Holland, Amsterdam (1996)

[2] G. R. Myneni, Physical and Mechanical Properties of Nb for SRF Science \& Technology, Single Crystal Niobium Technology Workshop CBMM, Araxa, Brazil 10/30-11/1, 2006

[3] R.E. Ricker, D.J. Pitchure, G.R. Myneni, The Effect of Interstitial Solutes on Deformation in Niobium and Niobium Single Crystals, Single Crystal Niobium Technology Workshop CBMM, Araxa, Brazil 10/30-11/1, 2006

[4] T. Gnaeupel-Herold, H.J. Prask, R.J. Fields, T.J. Foecke, Z.C. Xia, U. Lienert, $A$ Synchrotron Study of Residual Stresses in a Al6022 Deep Drawn Cup, Mat. Sci. Eng. A366 (2004), 104-113

[5] T. Foecke,T. Gnaupel-Herold, Robustness of the Sheet Metal Springback Cup Test, Metallurgical and Materials Transactions A, Volume 37, Number 12, December 2006, pp. 3503-3510(8)

[6] Hauk, V.(ed.), Structural and Residual Stress Analysis by Nondestructive Methods Evaluation, Application, Assessment, Elsevier, Amsterdam, 1997

[7] Reimers, W. (1992), Investigations of Large Grained Samples - Principles, in : H. T. Hutchings, A. D. Kravitz (Hrsg.), Measurement of Residual and Applied Stress Using Neutron Diffraction, Kluwer Academic Publishers, Dordrecht 1992, 159-170

[8] B. Spaniol, W.C. Heraeus GmbH and its activities regarding large grain Niobium Discs, Single Crystal Niobium Technology Workshop CBMM, Araxa, Brazil 10/30-11/1, 2006 